Article

\title{
A 3D Electroactive Polypyrrole-Collagen Fibrous Scaffold for Tissue Engineering
}

\author{
Soh-Zeom Yow ${ }^{1}$, Tze Han Lim ${ }^{2}$, Evelyn K. F. Yim ${ }^{3,4,5}$, Chwee Teck Lim ${ }^{3,4,6}$ and \\ Kam W. Leong ${ }^{2,7, *}$
}

1 Graduate Program in Bioengineering, Yong Loo Lin School of Medicine, National University of Singapore, 28 Medical Drive, Singapore 117456, Singapore;

E-Mail: sohzeom@gmail.com (S.-Z.Y.)

2 Duke-NUS Graduate Medical School Singapore, 2 Jalan Bukit Merah, Singapore 169547, Singapore; E-Mail: tzehan.lim@gmail.com (T.H.L.)

3 Division of Bioengineering, National University of Singapore, 9 Engineering Drive 1, Singapore 117576, Singapore; E-Mails: eyim@nus.edu.sg (E.K.F.Y.); ctlim@nus.edu.sg (C.T.L.)

4 Mechanobiology Institute, National University of Singapore, T-Lab, \#05-01, 5A Engineering Drive 1, Singapore 117411, Singapore

5 Department of Surgery, Yong Loo Lin School of Medicine, National University of Singapore, 1E Kent Ridge Road, Singapore 119228, Singapore

6 Department of Mechanical Engineering, National University of Singapore, 9 Engineering Drive 1, Singapore 117576, Singapore

7 Department of Biomedical Engineering, Duke University, Durham, NC 27708, USA

* Author to whom correspondence should be addressed; E-Mail: kam.leong@duke.edu; Tel.: +1-919-660-8466; Fax: +1-919-660-0031.

Received: 5 January 2011; in revised form: 6 February 2011 / Accepted: 24 February 2011 / Published: 28 February 2011

\begin{abstract}
Fibers that can provide topographical, biochemical and electrical cues would be attractive for directing the differentiation of stem cells into electro-responsive cells such as neuronal or muscular cells. Here we report on the fabrication of polypyrrole-incorporated collagen-based fibers via interfacial polyelectrolyte complexation (IPC). The mean ultimate tensile strength of the fibers is $304.0 \pm 61.0 \mathrm{MPa}$ and the Young's Modulus is $10.4 \pm 4.3 \mathrm{GPa}$. Human bone marrow-derived mesenchymal stem cells (hMSCs) are cultured on the fibers in a proliferating medium and stimulated with an external electrical pulse generator for 5 and 10 days. The effects of polypyrrole in the fiber system can be
\end{abstract}


observed, with hMSCs adopting a neuronal-like morphology at day 10, and through the upregulation of neural markers, such as noggin, MAP2, neurofilament, $\beta$ tubulin III and nestin. This study demonstrates the potential of this fiber system as an attractive 3D scaffold for tissue engineering, where collagen is present on the fiber surface for cellular adhesion, and polypyrrole is encapsulated within the fiber for enhanced electrical communication in cell-substrate and cell-cell interactions.

Keywords: polypyrrole; collagen; electrical stimulation; mesenchymal stem cells; fibrous scaffold; electroactive scaffold

\section{Introduction}

Stem cells can self-renew or differentiate depending on the culture microenvironment. While the exact regulatory mechanism of human stem cell differentiation remains to be fully elucidated, stem cells do respond to topographical and biochemical cues [1-4]. Nanotopographical cues and addition of retinoic acid have shown to induce various neural gene expressions in human mesenchymal stem cells [5]. Mechanical properties of the substrates, such as stiffness alone, can influence the differentiation of human mesenchymal stem cells (hMSC) into neuronal, muscular, or osteogenic lineages [6]. These studies point to a common observation, that the culture microenvironment of stem cells can direct or determine their fate. Therefore, where tissue engineering applications are concerned, a bio-interactive fibrous scaffold that can provide an appropriate microenvironment to direct stem cells to a desired differentiation lineage is an attractive option. It is not only a platform that permits the investigation of stem cell-microenvironment behavior, but also promotes the understanding of the mechanisms governing stem cell tissue regeneration.

Interfacial polyelectrolyte complexation is a fiber forming technique which occurs at the interface of aqueous solutions containing oppositely charged polyelectrolytes. Charge neutralization of the water-soluble polyelectrolytes at the interface, results in the self-assembled formation of an insoluble complex, which can take the form of a fiber if the as-formed complex is continuously removed from the interface by mechanical pinching and drawing. As the main fiber forms through the coalescence of several nascent fibers with smaller diameters, concomitant encapsulation of compounds dissolved in the polyelectrolyte solutions occurs readily. The entire process occurs at room temperature and being aqueous based, is well-suited for the encapsulation of biologics.

We have previously synthesized polyelectrolyte complexation (PEC) fibers from methylated collagen and synthetic terpolymer polyelectrolytes via interfacial polyelectrolyte complexation (IPC) for hMSC culture [7]. The ambient fiber drawing conditions avoid the denaturing of collagen and permit the formation of a bioactive PEC fiber. The retention of bioactivity is advantageous as the presentation of collagen in the fibers promotes cell attachment and viability. The PEC fiber system also permits the encapsulation of live hMSC, thus providing an excellent 3D platform to investigate stem cell-microenvironment behavior. We found that under the same culture conditions and time-point, the gene expression profiles of hMSC encapsulated in, or seeded on, the PEC fibers are different, indicating that the cellular microenvironment does influence stem cell differentiation. 
Despite their advantages, the collagen-terpolymer PEC fibers are mechanically weak. By incorporating polypyrrole (PPy) into the fibers, we hypothesize that it would improve their mechanical properties as well as introduce electroactivity to the fibrous scaffold. Polypyrrole is a well-studied biomaterial for numerous applications in tissue engineering [8-10]. The monomer pyrrole does not possess a net charge, therefore rendering it suitable for PEC fabrication. Once polymerized within PEC, the polypyrrole imparts electroactivity to the fibers. Conducting polymers derived from hetero-aromatic monomers such as pyrrole, thiophene, aniline and their derivatives have been gaining interest in tissue engineering. Such polymers consist of alternating double-and single-bonds that readily afford extensively conjugated structures through pi-overlap. Doping with anionic or cationic dopants imparts conductivity to these polymers [11]. In the presence of an electrical stimulation, these conductive polymers can modulate cell adhesion, migration, protein secretion and DNA synthesis of electrically responsive cells, such as nerve, bone, muscle and cardiac cells [12-21].

Polypyrrole has demonstrated cell and tissue compatibility in vivo and in vitro [12,22,23]. Human umbilical vein endothelial cells demonstrated higher cell doubling times when cultured on polypyrrole composite substrates [24]. Rat pheochromocytoma (PC-12) cells cultured on polypyrrole films in the presence of an electrical stimulus showed significant increase in neurite lengths [10]. Glia and primary neurons showed neurite extension and faster polarization on polypyrrole films. Fibronectin adsorption to polypyrrole increased under electrical stimulation [25] and led to enhanced neurite extension of nerve cells. Thus the presence of an electrical stimulus improves the quality of an engineered tissue, particularly in neural and muscular tissue regeneration.

Although numerous studies involving collagen-based scaffolds have been reported [7,26-28], there is scant information on the application of collagen in conjunction with conducting materials in tissue engineering. This discrepancy could be ascribed to the lack of viable methods for the preparation of the required hybrids of collagen and conducting polymers.

Li et al. [29] first attempted preparation of collagen/polypyrrole hybrids through polymerizing pyrrole dispersed in aqueous collagen solution with a $\mathrm{FeCl}_{3}$ initiator. However, formed polypyrrole was readily precipitated from solution and separated from collagen. They ascribed the difficulty of obtaining a hybrid to the incompatibility between the surface energies of polypyrrole and collagen. Ateh et al. [12] reported the preparation of collagen/polypyrrole hybrids through electrochemical polymerization. Unfortunately, 2-dimensional thin films are invariably produced with electrochemical polymerization. This limits the utility of such materials in tissue engineering, where 3-dimensional scaffolds, most notably sub-micron fibers, have demonstrated superiority [30].

In this study, we developed an IPC-based strategy that incorporates $\mathrm{FeCl}_{3}$-doped polypyrrole into a collagen-based PEC fiber for the creation of a 3D electroactive biofunctional fibrous scaffold. To address the incompatibility between the surface energies of PPy and collagen, we used 2-hydroxypropyl- $\beta$-cyclodextrin to entrap pyrrole monomers into its hydrophobic cavity and incorporated them into the PEC fibers. Subsequent polymerization within the PEC fibers led to a collagen-based electroactive fibrous scaffold that has the biological motif needed for cellular adhesion, 3D fibrous structure to mimic the native tissue architecture and polypyrrole bearing positively-charged bipolarons with chloride $\left(\mathrm{Cl}^{-}\right)$dopants. Substantial rearrangement and movement of bipolarons and $\mathrm{Cl}^{-}$dopants occur during electrical stimulation, possibly triggering significant ionic conductivity and optimized tissue regeneration via enhanced intercellular communications between seeded cells. Atomic Force Microscopy (AFM) confirmed the successful encapsulation of polypyrrole particles 
within the PEC fibers. The Young's modulus of the fibers increased significantly from $2.5 \pm 0.7 \mathrm{GPa}$ to $10.4 \pm 4.3 \mathrm{GPa}$ along with ultimate fiber tensile strength increment from $214.3 \pm 26.5 \mathrm{MPa}$ to $304.0 \pm 61.0 \mathrm{MPa}$. Seeding hMSCs on the polypyrrole-incorporated PEC fibers resulted in the early expression of MAP2 at day 5 and subsequent expressions of noggin, neurofilament and nestin markers at day 10. The electroactive microenvironment in this fiber system has also induced the upregulation and filament-like organization of synaptophysin along with neuronal-like morphology in hMSCs.

\section{Results and Discussion}

The following terms are used in this paper: ES PPy-PEC refers to hMSCs seeded on PPy-PEC fibers and electrically stimulated. PPy-n-PEC refers to hMSCs seeded on PPy-PEC fibers, not stimulated. ES PEC refers to hMSCs seeded on PEC fibers, electrically stimulated. n-PEC refers to hMSCs seeded on PEC fibers and not stimulated.

\subsection{Fabrication of PPy-PEC Fibers}

Figure 1(a) shows the formation of 2-hydroxypropyl- $\beta$-cyclodextrin-pyrrole inclusion complex for the incorporation of pyrrole into PEC fibers. The inclusion complex was added to the anionic polyelectrolyte droplet and placed next to the cationic polyelectrolyte droplet before a PEC fiber was drawn from the interface in Figure 1(b). The pyrrole-containing inclusion complexes were encapsulated within the newly formed fiber as it was drawn away from the interface. The encapsulated pyrrole-containing complexes were polymerized with $0.1 \mathrm{M} \mathrm{FeCl}_{3}$ to form polypyrrole-PEC fibers.

Figure 1. (a) 2-Hydroxypropyl- $\beta$-cyclodextrin-pyrrole inclusion complex. (b) Fabrication of polypyrrole-incorporated PEC fibers.

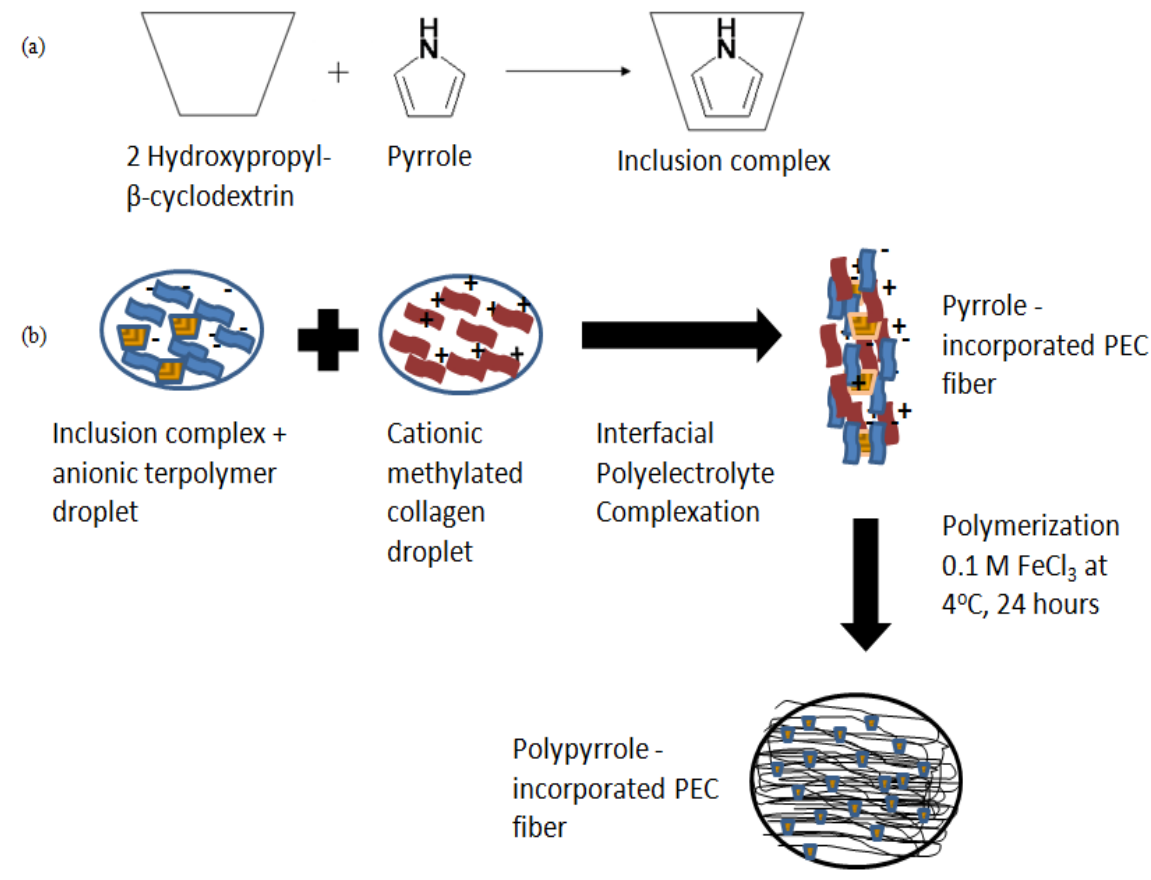

\subsection{AFM Studies of Fiber Surface Morphology}

The fiber samples were first observed with an optical microscope. The regions where polypyrrole was present in the fibers were seen clearly under polarized light in Figure 2(a). Polypyrrole particles 
were found dispersed in the core fiber as well as the fiber bead regions. AFM images of the polypyrrole regions show the conservation of the fiber surface topography as evidenced by the height and amplitude images, which are similar to those of fiber regions without polypyrrole. The difference lies with the AFM phase images, in which the polypyrrole regions show distinct material phases, where the bright regions correspond to areas with higher modulus and the darker regions correspond to areas with relatively softer material modulus. AFM phase images of the regions without polypyrrole, on the other hand, do not show phases with such distinct material modulus.

Figure 2. (a) AFM imaging of PPy-PEC fibers showing the height, amplitude and phase images of polypyrrole regions and fiber regions (where polypyrrole particles were absent).The obtained images are compared with the optical image of the scanned fiber regions (indicated by the boxes and arrows). AFM scan bars for polypyrrole regions and fiber regions are $2 \mu \mathrm{m}$ and $1 \mu \mathrm{m}$ respectively. (b) AFM imaging and section analysis of polypyrrole particles that were incorporated in PPy-PEC fibers. The AFM scan bar of the height and amplitude images is $10 \mu \mathrm{m}$. (c) Mechanical properties of PPy-PEC and PEC fibers (mean $\pm \mathrm{SD}, \mathrm{n}=10$ ).

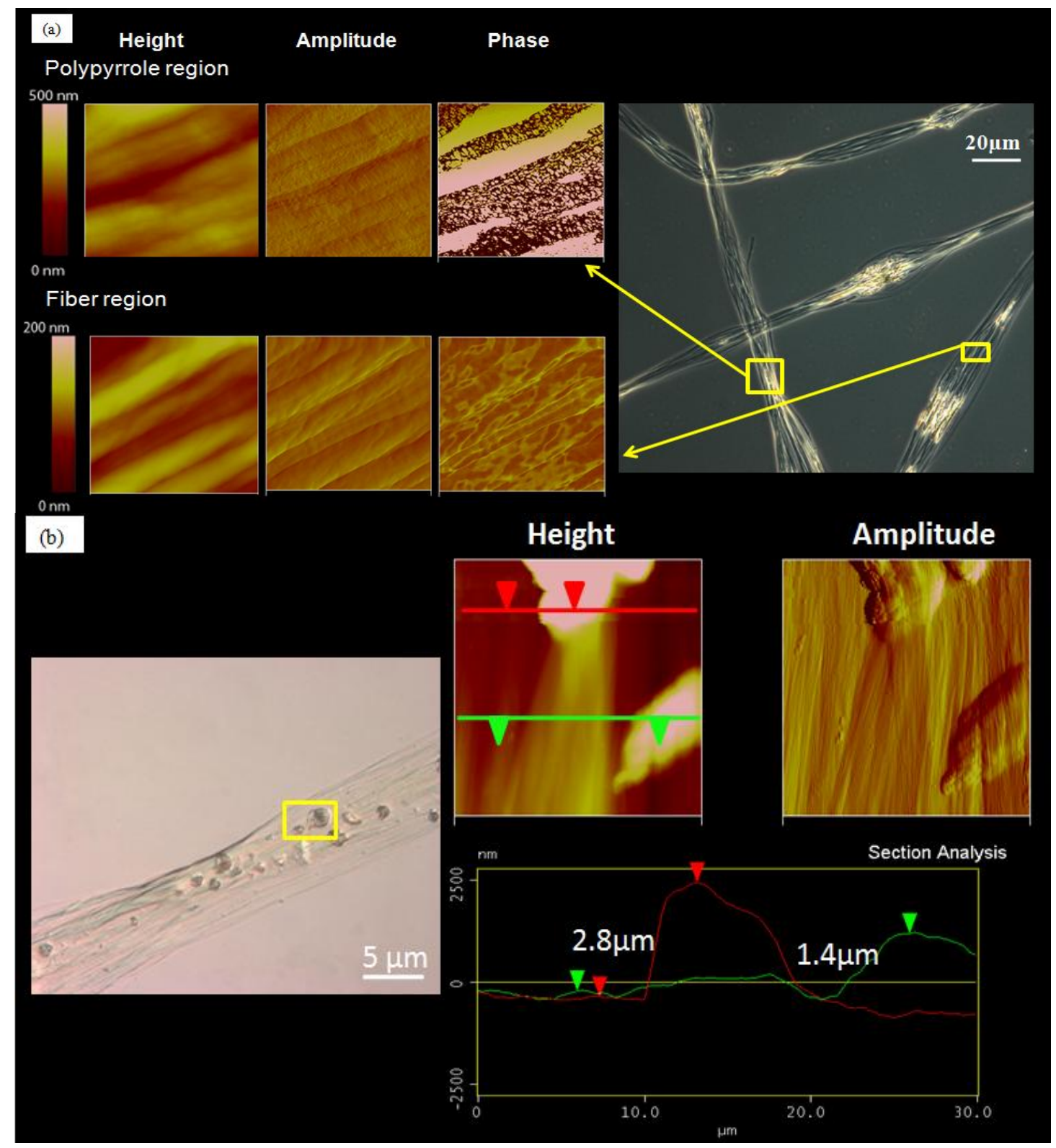


Figure 2. Cont.

(c)

\begin{tabular}{lllll}
\hline Fiber Composition & $\begin{array}{l}\text { Tensile strength } \\
(\mathrm{MPa})\end{array}$ & $\begin{array}{l}\text { Strain at } \\
\text { failure }(\%)\end{array}$ & $\begin{array}{l}\text { Young's } \\
\text { Modulus (GPa) }\end{array}$ & $\begin{array}{l}\text { Toughness } \\
(\mathrm{MPa})\end{array}$ \\
\hline $\begin{array}{l}\text { Polypyrrole-Collagen } 5 \mathrm{mg} / \mathrm{mL}- \\
\text { Terpolymer } 0.55 \mathrm{wt} \%\end{array}$ & $304.0 \pm 61.0$ & $9.91 \pm 4.7$ & $10.4 \pm 4.3$ & $15.9 \pm 8.8$ \\
& & & & \\
\hline $\begin{array}{l}\text { Collagen } 5 \mathrm{mg} / \mathrm{mL}-\text { Terpolymer } \\
0.55 \mathrm{w} \text { \% }\end{array}$ & $214.3 \pm 26.5$ & $21.8 \pm 5.6$ & $2.5 \pm 0.7$ & $20.0 \pm 0.1$ \\
\hline
\end{tabular}

AFM section analysis was carried out on scanned images of polypyrrole regions and the results are presented in Figure 2(b). From the AFM images, the polypyrrole particles were incorporated within the fibers and not coated onto the fiber surface. This is evidenced by the presence of aligned sub-micron fibrils encapsulating the polypyrrole particles. Section analysis of the incorporated polypyrrole particles measures the size of the incorporated particles by determining the vertical distances between the substrate and the tip of polypyrrole particles, indicated by the red and green arrows. The size of the polypyrrole particles was in the range of 1.4 to $3.0 \mu \mathrm{m}$.

The results show that the fiber bead regions tend to be regions concentrated with the "encapsulants" of interest. The fiber bead regions are rich in polypyrrole, suggesting that pyrrole monomers tend to congregate in this region before polymerization occurs.

\subsection{Mechanical Property of PPy-PEC Fibers}

The effects of polypyrrole encapsulation on the mechanical properties of the fibers are presented in Figure 2(c). The Young's modulus of PEC fibers was 2.5 $\pm 0.7 \mathrm{GPa}$ and increased to $10.4 \pm 4.3 \mathrm{GPa}$ when polypyrrole was embedded and polymerized to polypyrrole in the fibers. The tensile strength of the fibers was also higher when polypyrrole particles were present within the fiber.

\subsection{Cell Viability and mRNA Expression}

hMSCs were seeded on PPyPEC fibers and subjected to electrical stimulus for 5 and 10 days (ES PPy-PEC fibers) while another set of PPyPEC fibers was cultured under the same conditions concurrently without electrical stimulus (PPy-n-PEC) as presented in Figure 3(a). With electrical stimulus, hMSC proliferation at day 5 was higher than the non stimulated counterpart $(\mathrm{p}<0.01)$. However, at day 10 , the reverse was observed $(\mathrm{p}<0.005)$, suggesting that prolonged electrical stimulus on hMSCs grown under these culture conditions may have a detrimental effect on their viability and proliferation. 
Figure 3. (a) hMSC viability and proliferation results at day 5 and day 10. Statistical significance: * $\mathrm{p}<0.0001$ (between ES PPy-PEC and PPy-n-PEC samples at day 5), ** $\mathrm{p}<0.005$ (between ES PPy-PEC and PPy-n-PEC samples at day 10) (b) mRNA expressions of hMSCs at day 5 and day 10. Control refers to hMSCs seeded on glass coverslips.

(a)

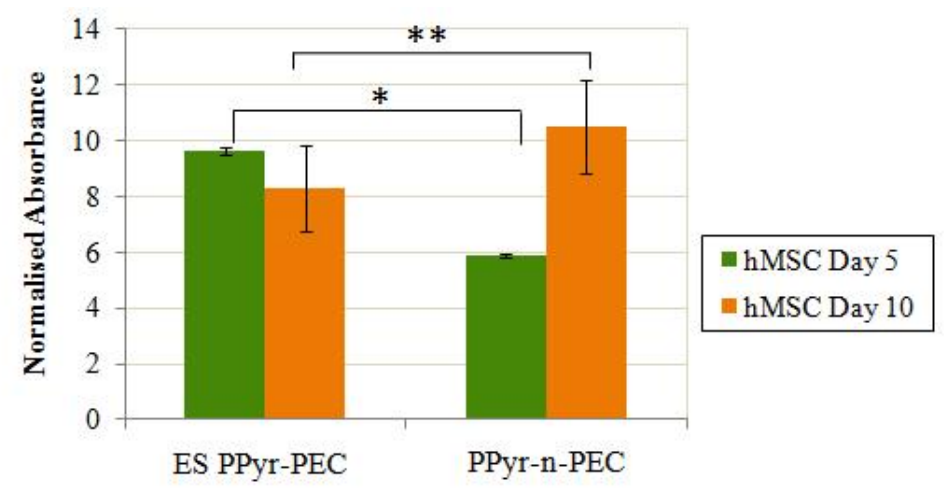

(b)

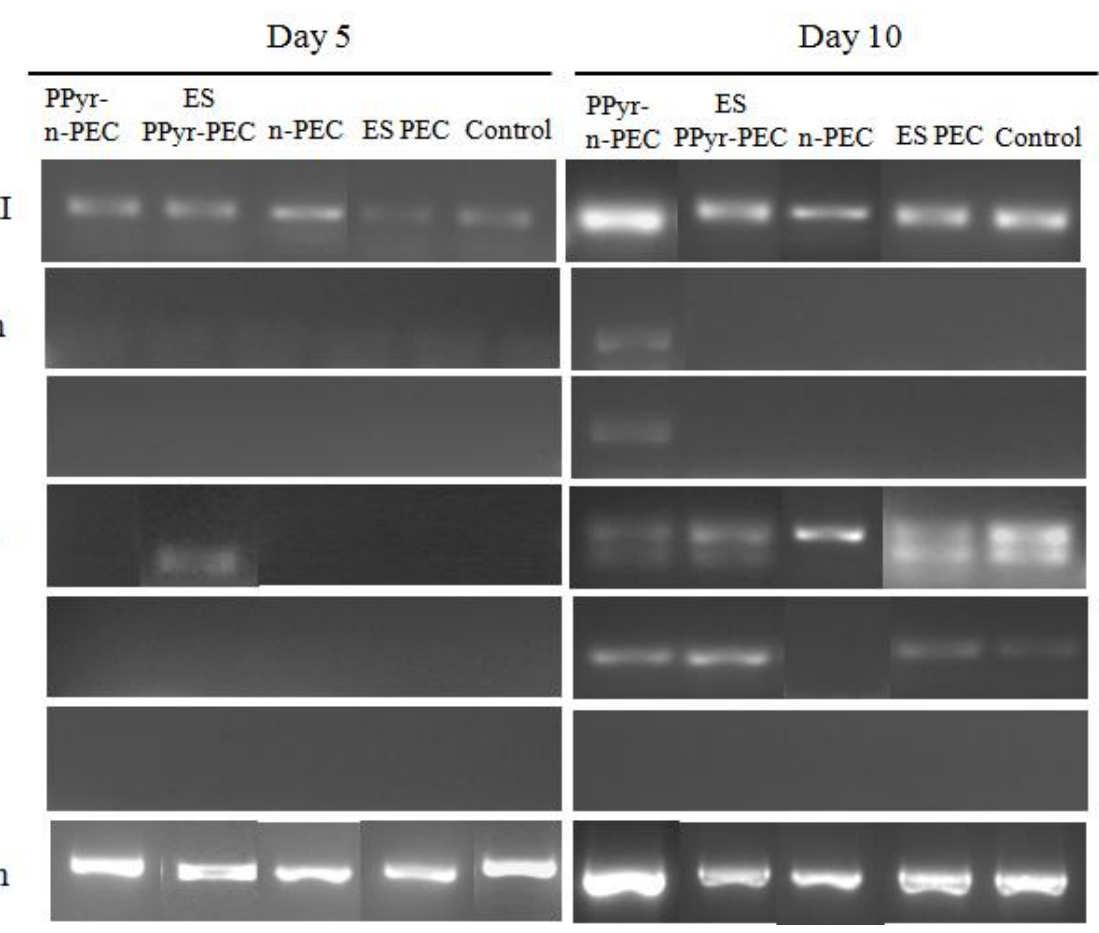

The expressions of neural markers in hMSCs seeded on PPy-PEC and PEC fibers are shown in Figure 3(b). $\beta$ tubulin III was present in all samples while GFAP expression was absent. At day 5, MAP2 was expressed in ES PPy-PEC samples, but absent in ES PEC, PPy-n-PEC, n-PEC and control samples. The results suggest that the presence of polypyrrole in the fibers together with the application of an external electrical stimulus provided the appropriate conditions for MAP2 expression by day 5 . By day 10, MAP2 was expressed in all samples.

At day 10, nestin was expressed by hMSCs in ES PPy-PEC and PPy-n-PEC samples. The expression was comparatively weaker in ES PEC and control samples. Nestin expression was absent in n-PEC samples. Noggin and neurofilament were only expressed in PPy-n-PEC fibers at day 10. Both ES PPy-PEC and ES PEC samples showed similar mRNA expression profiles as the control samples. 


\subsection{Immunostaining of Neural Genes and Proteins}

Figure 4 shows the immunostaining of $\beta$ tubulin III, MAP2 and connexin 43 in hMSCs samples on day 5 and day 10. At day 5, connexin 43, a gap junction protein was expressed in all samples but the expression was very weak in PPy-n-PEC samples (Figure 4(a)). $\beta$ tubulin III was detected in all samples for both days. At day 5, MAP2 protein expression was highest in hMSCs cultured on PPy-n-PEC and ES PPy-PEC fibers. The expression was very weak in controls. By day 10, MAP2 expression was downregulated in ES PPy-PEC samples and upregulated in PPy-n-PEC and control samples (Figure 4(b)).

Figure 4. (a) Immunofluorescent staining of $\beta$ tubulin III, MAP 2 and connexin 43 in hMSCs cultured on ES PPy-PEC, PPy-n-PEC and on control samples at day 5. (b) Immunofluorescent staining of $\beta$ tubulin III and MAP 2 in hMSCs cultured on ES PPy-PEC, PPy-n-PEC, ES PEC and control samples at day 10. Scale bar $=20 \mu \mathrm{m}$. The cell nuclei in all panels were stained with DAPI and shown in blue.

(a)

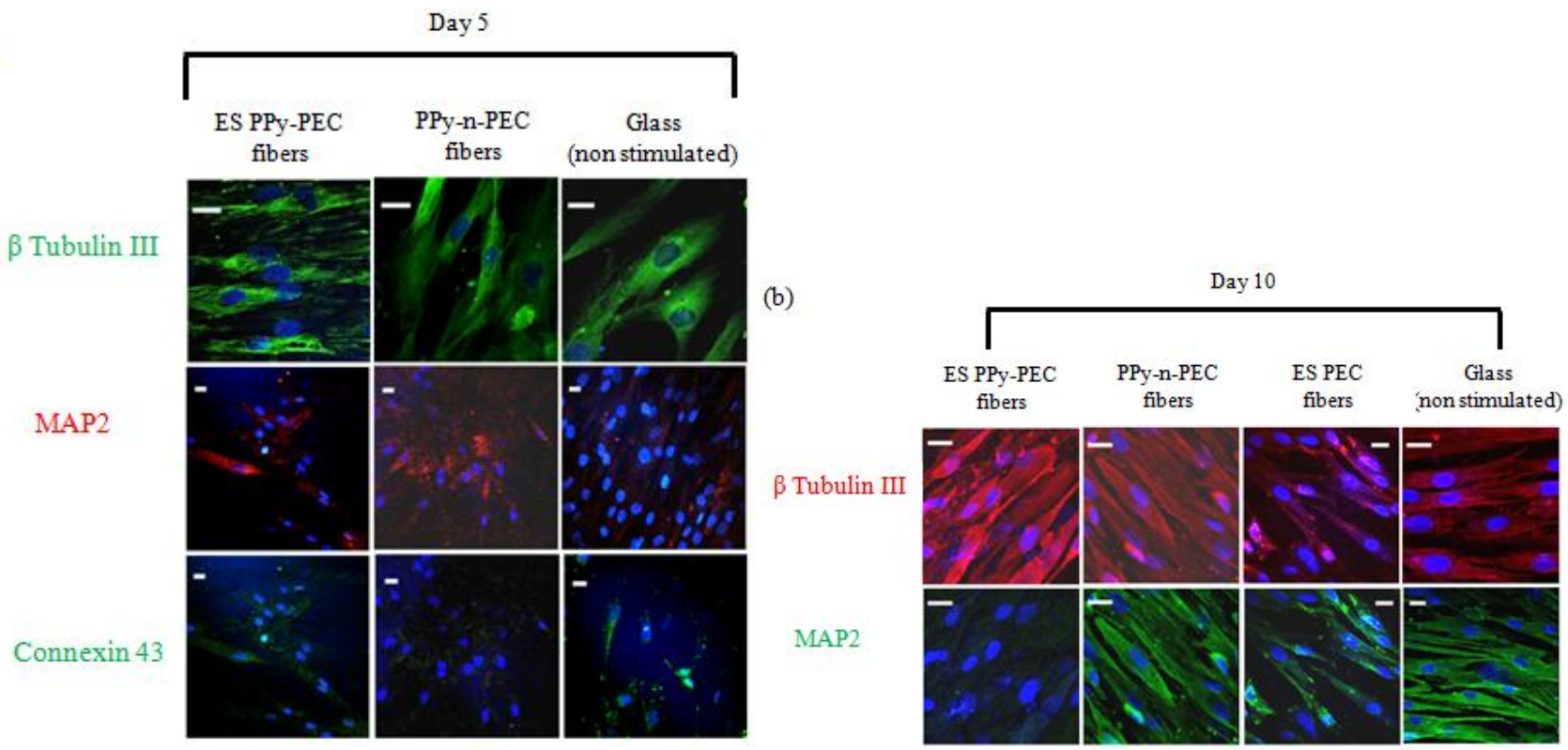

\subsection{1. $\beta$ Tubulin III and MAP 2 Expression in hMSCs}

To determine if the changes in neuronal marker MAP2 expression was due to the material or prolonged electrical stimulation or a combination of both, hMSCs in ES PEC samples were cultured and stimulated in the same conditions for 10 days and the images were compared with ES PPy-PEC, PPy-n-PEC and control samples in Figure 4(b).

$\beta$ tubulin III and MAP2 expressions were evident in ES PEC samples although they were not as highly expressed as those in PPy-n-PEC and control samples. In the case of ES PPy-PEC samples, where the culture conditions were exactly the same but hMSCs were seeded on polypyrrole-incorporated PEC fibers, the significant downregulation of MAP2 expression at day 10 suggests that prolonged electrical stimulation and the presence of polypyrrole particles in the system may have inhibited the hMSC differentiation. 


\subsubsection{Synaptophysin Expression in hMSCs}

At day 5, synaptophysin was highly expressed and organized into filaments in ES PPy-PEC samples (Figure 5), suggesting that hMSCs were responding to the electrical stimulus and forming synapse connection. The synaptophysin expression in PPy-n-PEC, n-PEC, ES PEC and control samples was significantly weaker and the filament-like organization was absent.

Figure 5. Immunofluorescent staining of synaptophysin in ES PPy-PEC, PPy-n-PEC, ES PEC, n-PEC and control samples at day 10. Control is hMSCs seeded on glass coverslips in the absence of electrical stimulation. Synaptophysin was stained with either Alexa Fluor 488 anti goat (green) or Alexa Fluor 568 anti rabbit (red) secondary antibodies. The cell nuclei in all panels were stained with DAPI and shown in blue.

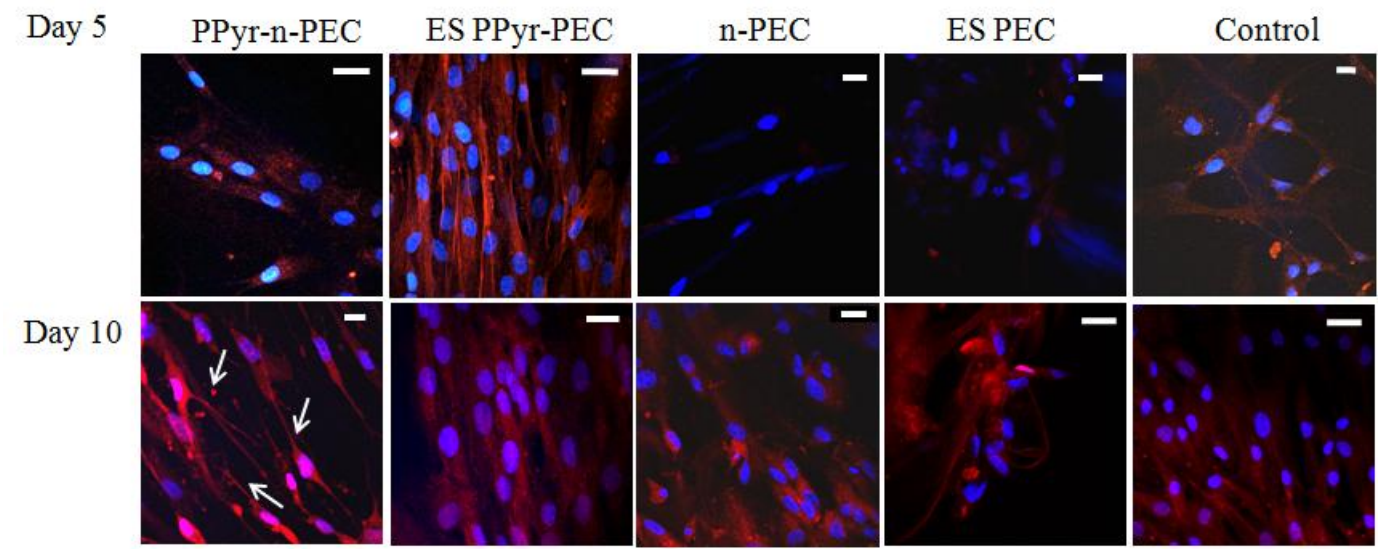

At day 10, the filament-like organization of synaptophysin in ES PPy-PEC samples was not as evident as in day 5 while the hMSCs in PPy-n-PEC samples showed synaptophysin upregulation and the cells adopted neuronal-like morphology, as indicated by the arrows in Figure 5. hMSCs in n-PEC, ES PEC and control samples expressed synaptophysin but did not adopt neuronal-like morphology.

\subsection{Discussion}

Wan et al. [31] pioneered the use of PEC fibers of sodium alginate and chitosan polyelectrolytes as TE scaffolds. These scaffolds however, displayed poor cellular adhesion and uneven cell distribution. Additionally, poor mechanical properties necessitated post-fabrication treatment in the form of a sol-gel procedure. PEC fibers derived from methylated collagen and deprotonated terpolymer $(2: 1: 1)$ of methyl methacrylate (MMA), 2-hydroxyethyl methacrylate (HEMA) and methacrylic acid (MAA) were prepared by Yow et al. [7] to address the shortfalls of the earlier scaffolds. Integrin-rich collagen enhanced cellular interactions while terpolymer components afforded requisite physical properties. For instance, mechanical strength was afforded from MMA, water-uptake and aqueous exchange from HEMA and negative charges for complexation from MAA. Additionally, PEC fibers with tunable physical properties may be readily afforded through varying monomer proportions in the terpolymer. IPC-derived (PEC) fibers as such, are akin to traditional polyelectrolyte multilayers (PEMs) prepared with layer-by-layer deposition techniques. In contrast to PEMs, where self-assembly and encapsulation occurs on a given substrate through tedious sequential coatings, IPC fiber formation and encapsulation 
is advantageous as a facile one-pot process and requiring no supporting substrate. Unlike PEMs however, there are presently no reports of PEC fibers containing incorporated polypyrrole. This work constitutes the first instance of polypyrrole incorporated PEC fibers and was made possible through the use of suitable cyclodextrins to facilitate successful encapsulation of hydrophobic pyrrole into hydrophilic PEC fibers during IPC. This same strategy could potentially enable the hitherto unfeasible encapsulation of a range of hydrophobic species including monomers and quantum dots into PEC fibers.

\subsubsection{Polypyrrole Incorporation into PEC Fibers}

The appeal of the proposed fibrous material in this study comes from having a 3-D high surface area for cell-cell contact; collagen to offer biochemical cues for cell adhesion, and ferric chloride doped polypyrrole for electroactivity. Initial attempts to incorporate polypyrrole directly into fibers by IPC have proved unsuccessful. In such attempts, pyrrole was first dispersed into aqueous solutions containing up to $0.5 \mathrm{wt} \%$ of terpolymer to form a suspension followed by complexation with aqueous solutions of methylated collagen for subsequent fiber drawing before immersing in the $\mathrm{FeCl}_{3}$ polymerization bath overnight. The extent of polypyrrole formation within the fibers was negligible and this could be due to the poor uptake of pyrrole into the fibers during IPC, which in turn, could be ascribed to the hydrophobic nature and high vapor pressure of pyrrole. As PEC fibers are formed at the interface where high density of opposite charges is essential for fiber formation and coalescence to occur [32], water soluble, hydrophilic compounds are readily encapsulated within these regions. In contrast, hydrophobic compounds such as pyrrole would be readily expelled from the fiber forming regions with consequentially lower levels being made available for polymerization. In addition, the high vapor pressure of pyrrole led to significant loss by evaporation.

2-hydroxypropyl- $\beta$-cyclodextrin is selected as the cyclodextrin "host" in this work because of its excellent solubility in aqueous media and low cytotoxicity [33]. The ability of $\beta$-cyclodextrin to encapsulate lipophilic compounds, such as pyrrole, in its hydrophobic activity to form a water-soluble inclusion complex (IC) is well documented [34]. IC formation can significantly lower the vapor pressure of pyrrole. Hence, pyrrole/ $\beta$-cyclodextrin ICs represents a strategy by which pyrrole may be effectively encapsulated within IPC fibers for subsequent polymerization. Additionally, chemical polymerization by $\mathrm{FeCl}_{3}$ of cyclodextrin encapsulated pyrrole has been reported to precipitate polypyrrole as a powder, with cyclodextrin "hosts" retained in solution from which they may be efficiently separated from polypyrrole by sedimentation and washing with water [34].

\subsubsection{Fiber Characterization}

AFM imaging of PEC fibers reveals that each PEC fiber is a bundle of sub-micron fibrils orientated along the fiber axis, and the fibrils are mainly made up of distinct material phases [7]. The polypyrrole incorporation methodology which we have adopted enables the effective encapsulation of pyrrole monomers in PEC fibers and as the PEC fibers are permeable, pyrrole polymerization occurs readily when the fibers are immersed in aqueous iron (III) chloride solution overnight. AFM imaging confirms the presence of polypyrrole particles dispersed throughout the fibers. Hence, the fiber system would be particularly useful in tissue engineering applications, where collagen needs to be present on the fiber 
surface to aid cell adhesion, and polypyrrole particles are present within the fibers to allow for electrical stimulation of the adhered cells.

In the mechanical evaluation of single strands of PPy-PEC fibers, the decrease in strain at failure in the fibers is expected as the incorporation of polypyrrole particles, which have a higher material modulus than the PEC fiber matrix (softer modulus), leads to a material interface mismatch and results in highly stressed regions. When a tensile stress is applied to the fibers, the stress concentration at the polypyrrole and PEC interface may lead to crack formation and propagation and thus resulting in rapid fiber failure. This finding is in agreement with R.A. Green's observation [16] that addition of an electroactive component into a material often increases the material brittleness.

\subsubsection{Expression of Neural Markers in hMSCs}

MAP2 plays an essential role in determining and stabilizing the dendritic cell shape during neuron development. We observed in our previous study [7] that MAP2 mRNA expression on PEC fibers is usually detected from day 7, when the cells start to align along the PEC fibers. When seeded on ES PPy-PEC samples, MAP2 expression is detected by day 5, while hMSCs seeded on PPy-n-PEC and ES PEC samples do not express MAP2 at the same time point (Figure 3(b)). This observation suggests that the presence of an external electrical stimulus together with the polypyrrole particles in the fiber matrix could have provided a microenvironment that promotes the early expression of MAP2 in hMSCs.

Synaptophysin is a synaptic vesicle glycoprotein found in neurons and it participates in synaptic transmissions. The protein expression in ES PPy-PEC samples shows filament-like organization at day 5. Such organization of the protein is not detected in PPy-n-PEC and ES PEC samples at the same time point. The cell-to-cell synaptic transmission appeared enhanced in ES PPy-PEC samples which, together with the observation in MAP2 expressions; demonstrates the synergic combination of a polypyrrole-incorporated fiber matrix and an external electrical stimulus in inducing hMSCs to express neural genes.

However, this stimulus needs moderation, as the continuous external electrical stimulus harms the proliferation and viability of the attached hMSCs, accompanied by a down-regulation of the MAP2 protein expression. Although the immunofluorescent staining of MAP2 expression in ES PEC samples is weaker than the PPy-n-PEC samples, the results collectively suggest that the incorporation of polypyrrole in the fibers or the use of external electrical stimulation alone does not cause the severe downregulation of the neuronal marker in ES PPy-PEC samples. Instead, it suggests that prolonged electrical stimulation is detrimental to the hMSCs and the adverse impact is more pronounced when polypyrrole particles are present in the fiber matrix to enhance the electrical signaling within the cells. The loss of filament-like organization of synaptophysin in ES PPy-PEC samples at day 10 further cements our speculation on the possibility of overstimulating the hMSCs. The electrical stimulation parameters need to be optimized further as it is evident that stimulation of cultures at the early stage of culture does encourage the hMSCs to form synaptic transmissions and express neuronal-specific markers. However the extent of stimulation has to be moderated to avoid introducing cell stress and compromising cell viability. 
At day 10, synaptophysin expression of hMSCs in PPy-n-PEC samples has increased compared to day 5 and the cells have adopted a morphology that is similar to that of neurons in Figure 5. Noggin and neurofilament (NFL) mRNAs, both markers of neural specific cells are also expressed in PPy-n-PEC samples. The cell morphology and expression of these markers are absent in n-PEC samples under the same culture conditions. These observations collectively suggest that polypyrrole particles in the fiber matrices are capable of interacting and mediating the electrical signaling process within the seeded hMSCs.

Our proposed strategy of encapsulating pyrrole into PEC fibers via the inclusion complex and subsequent in situ polymerization with iron (III) chloride proves effective in the fabrication of 3D electroactive, collagen-based fibers. Favorable adhesion of hMSCs on the fibers is evident and possibility of cell-cell electrical signaling is also demonstrated. With optimization of the electrical stimulation and encapsulation of growth factors into the PPy-n-PEC fibers, one can envision the potential of this system in providing a more conducive microenvironment for the differentiation of different sources of stem cells into the neuronal, muscle, or cardiac lineages.

\section{Experimental Section}

The terpolymer and methylated collagen were synthesized as described previously [7]. Briefly, terpolymer of methyl methacrylate (MMA), hydroxyethyl methacrylate (HEMA) and methacrylic acid (MAA) was synthesized via free radical polymerization and deprotonated with $0.1 \mathrm{M} \mathrm{NaOH}$ to afford an anionic terpolymer polyelectrolyte. Collagen was methylated using methanol containing $0.1 \mathrm{M} \mathrm{HCl}$ to form a cationic methylated collagen polyelectrolyte. Pyrrole monomers were purified by vacuum distillation before use.

\subsection{Polypyrrole-Incorporated PEC (PPy-PEC) Fiber Fabrication}

$1.18 \mathrm{~g}$ of 2-hydroxypropyl- $\beta$-cyclodextrin was dissolved in $10 \mathrm{~mL}$ of ultrapure water and kept on the shaker for 30 minutes. $70 \mu \mathrm{L}$ of neat pyrrole was added into the solution to produce a pyrrole concentration of $0.1 \mathrm{M}$. The 2-hydroxypropyl- $\beta$-cyclodextrin-pyrrole mixture was kept on the shaker for 1 hour before adding it to $10 \mathrm{~mL}$ of terpolymer and vortexed.

Pyrrole-incorporated PEC fibers were made by drawing them out of the interface between pyrrole-terpolymer and methylated collagen droplets. The fibers were collected immediately and placed in a petri dish of $0.1 \mathrm{M}$ of $\mathrm{FeCl}_{3}$ for 24 hours at $4{ }^{\circ} \mathrm{C}$. The fibers were washed in $100 \%$ ethanol and deionized water twice and dried in a dessicator overnight.

For cell culture studies, the fibers were placed on $15 \mathrm{~mm}$ glass cover slips and sterilized by soaking in $70 \%$ ethanol for 30 minutes before 3 hours of UV irradiation. The samples were washed 3 times with sterile PBS before use.

\subsection{PPy-PEC Fiber Fabrication}

\subsubsection{Fiber Surface Morphology Investigation}

AFM imaging was used to examine the surface morphology of the PPy-PEC fibers. Briefly, the fibers were collected on mica substrates, washed twice in $100 \%$ ethanol and ultrapure water and dried 
in a desiccator for 12 hours before imaging in tapping mode. Silicon nitride tips with resonance frequencies of $289-293 \mathrm{kHz}$ were used to scan the fiber surface.

\subsubsection{Mechanical Property Measurement}

The tensile properties of the fibers were measured with a nano tensile testing system (Nano Bionx Systems, MTS, USA) at room temperature and in dry condition. Briefly, single strands of polypyrrole-PEC and PEC fibers were individually mounted onto a cardboard frame. The frame bearing a single fiber strand was clamped onto the nano tensile tester and subjected to uniaxial tensile loading at a strain rate of $3.7 \times 10^{-2} \mathrm{~s}^{-1}$ until breakage. Each fiber had a gauge length of $10 \mathrm{~mm}$.

\subsection{Electrical Stimulation Setup}

A cell culture electrical stimulation setup was built in-house. The cover lid of a 6-well culture plate was removed and slits of size $0.5 \mathrm{~mm}$ on the lid were drilled, as shown in Figure 6. Rectangular platinum (Pt) strips with dimensions $25 \mathrm{~mm} \times 0.1 \mathrm{~mm} \times 28 \mathrm{~mm}$ (Alfa Aesar, USA) were placed through the slits and fixed in place with medical grade silicone adhesive. When dried, the cover plate bearing the Pt strips were checked to make sure that the Pt strips were firmly in place with no gaps between the strips and the slits. Copper wires were soldered on the top of each Pt strips to connect the wells in series. The setup was hooked to the signal generator and a multimeter was used to make sure there were no loose or disconnected wires and Pt strips. The cover lid was sterilized by soaking in $70 \%$ ethanol overnight and left to dry in a biosafety cabinet before use.

Figure 6. (a) Setup of hMSC electrical stimulation device. A 6-well culture plate cover was modified so Pt strips could be slotted through the cover plate and fixed to deliver electrical pulses into each of the cell wells. The 6-well culture plate was connected to the function generator and stimulated in the cell culture incubator. (b) Close-up image of each well. The fiber sample, secured by a Teflon ring to the well, was placed between the Pt electrodes.

(a)

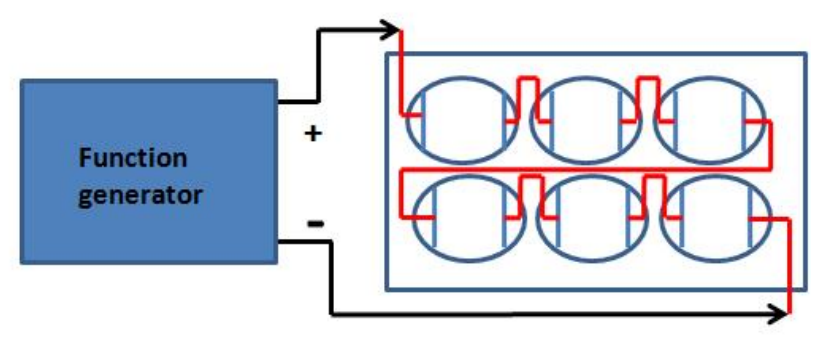

(b)

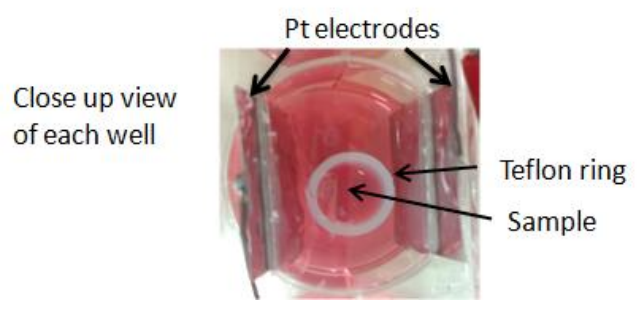

The fiber samples were placed in each well, secured with a Teflon ring and an electrical pulse was passed through the well through the Pt strips and cell culture medium to stimulate the cells seeded on 
the fiber samples. The pulse generator (HP function generator 33120A, Singapore) was programmed to generate square biphasic waves of $5.2 \mathrm{~V}$ to the entire setup so that square waveform electrical pulses of $1.2 \mathrm{~V}$ with pulse duration of $5 \mathrm{~ms}$ was delivered into each well at a frequency of $200 \mathrm{~Hz}$. The electrical stimulation setup was connected to an oscilloscope to ensure electrical pulses delivered to the cell wells were of the mentioned pulse duration, frequency and voltage before commencement of the cell culture studies.

\section{4. hMSC Cell Culture}

hMSCs between passages 4 to 7 were used for this study. The cells were cultured in mesenchymal stem cell growth medium (Poietics, Lonza, Switzerland, MSCGM ${ }^{\mathrm{TM}}$ ), trypsinized from T25 tissue culture flasks and $200 \mu \mathrm{L}$ of cell suspension containing about 7,000 cells were added to each fiber sample for 30 minutes. A Teflon ring was placed on each of the fiber sample to keep the sample in place and $4.8 \mathrm{~mL}$ of fresh growth medium was added to the 6 well culture plate and the samples were incubated at $37{ }^{\circ} \mathrm{C}$ with $5 \% \mathrm{CO}_{2}$. After 24 hours of cell seeding, the 6-well cell culture plate was replaced with the cover plate with platinum foils, connected to the function generator and electrically stimulated for 5 and 10 days. The cell culture medium was changed every day. Another set of hMSCs seeded on polypyrrole-incorporated PEC fibers was prepared and hMSCs seeded on glass cover slips were used as control. They were prepared in the same manner as described above but without electrical stimulation during culture.

\subsection{Cell Proliferation Assay}

The cell viability and proliferation of the cells were verified by Alamar Blue assay. At day 5 and day 10 of electrical stimulation, the fibers containing hMSCs were incubated with $400 \mu \mathrm{L}$ of cell culture medium containing $40 \mu \mathrm{L}$ of Alamar Blue solution for 3 hours. The medium was removed and fluorescence intensity readings were taken at excitation and emission wavelengths of $544 \mathrm{~nm}$ and $590 \mathrm{~nm}$ respectively. The readings were normalized with readings obtained from blank controls.

\subsection{Immunostaining of Neural Markers in hMSCs}

The samples were fixed with $4 \%$ paraformaldehyde for 20 minutes, permeabilized in $0.1 \%$ Triton X-100 for 20 minutes and blocked with 3\% BSA for 1 hour at room temperature. The hMSCs samples were stained sequentially with rabbit anti- $\beta$ tubulin III (Sigma Aldrich, Singapore) and goat anti-MAP 2 (Santa Cruz Biotechnology, USA) antibodies, rabbit anti-synaptophysin and goat anti-connexin 43. Alexa Fluor 488 and 568 anti-goat and Alexa Fluor 488 and 568 anti-rabbit secondary antibodies (Invitrogen, Singapore) were used for this study.

The samples were incubated with the first primary antibody at a dilution of 1:50 overnight at $4{ }^{\circ} \mathrm{C}$ and washed 3 times with $0.05 \%$ Triton X-100 in PBS. The first secondary antibody (either Alexa Fluor anti-goat or anti-rabbit secondary) was added to the samples at a dilution of 1:200 and incubated for 1 hour at room temperature in the dark. The samples were blocked with 3\% BSA for 1 hour in the dark at room temperature and were incubated with the second primary antibody (1:50 dilution). They were washed 3 times before incubation with secondary antibody for 1 hour in the dark and 
washed 3 times for 5 minutes each. All samples were counterstained with DAPI (1:2,500 dilution), mounted and viewed under a confocal microscope.

\section{7. mRNA Expression}

hMSCs samples were stabilized with RNAlater RNA stabilization Reagent (Qiagen, Singapore) and homogenized with QIAshredder. The RNA was isolated with RNeasy Minikit (Qiagen, Singapore). Synthesis and amplification of cDNA was carried out with Qiagen One-step RT-PCR kit with primers listed in Table 1 at an annealing temperature of $62{ }^{\circ} \mathrm{C} .38$ cycles of PCR were carried out on a MJ Research PTC 100 Thermal Cycler and the PCR products were resolved in $1.2 \%$ agarose gel in $1 \times$ TAE buffer $(75 \mathrm{~V}$ at $10 \mathrm{~cm})$.

Table 1. List of primers used in mRNA expression studies.

\begin{tabular}{|c|c|c|c|c|}
\hline Marker name & Accession number & Primer & Size bp & Sequence $\left(5^{\prime}-3^{\prime}\right)$ \\
\hline Noggin & NM_005450 & $\begin{array}{l}\text { Forward } \\
\text { Reverse }\end{array}$ & 247 & $\begin{array}{l}\text { AAAGGATCTGAACGAGACGCTGCT } \\
\text { CTTCTTGCTTAGGCGCTGCTTCTT }\end{array}$ \\
\hline$\beta$-tubulin III & NM_006086 & $\begin{array}{l}\text { Forward } \\
\text { Reverse }\end{array}$ & 144 & $\begin{array}{l}\text { ATGTGGTGCGGAAGGAGTGTGAAA } \\
\text { TGTTCATGATGCGGTCGGGATACT }\end{array}$ \\
\hline MAP2 & NM_002374 & $\begin{array}{l}\text { Forward } \\
\text { Reverse }\end{array}$ & 306 & $\begin{array}{l}\text { TAACCAACCACTGCCAGACCTGAA } \\
\text { GCCACATTTGGATGTCACATGGCT }\end{array}$ \\
\hline Neurofilament (NFL) & NM_006158 & $\begin{array}{l}\text { Forward } \\
\text { Reverse }\end{array}$ & 384 & $\begin{array}{l}\text { АCСТCСТCAACGTGAAGATGGCTT } \\
\text { АСТСТTCСTTGGCAGCTTCTTCCT }\end{array}$ \\
\hline Nestin & NM_006617 & $\begin{array}{l}\text { Forward } \\
\text { Reverse }\end{array}$ & 200 & $\begin{array}{l}\text { GCCCTGACCACTCCAGTTTA } \\
\text { GGAGTCCTGGATTTCCTTCC }\end{array}$ \\
\hline GFAP & NM_002055 & $\begin{array}{l}\text { Forward } \\
\text { Reverse }\end{array}$ & 199 & $\begin{array}{l}\text { ACCAGGACCTGCTCAATGTC } \\
\text { ATCTCCACGGTCTTCACCAC }\end{array}$ \\
\hline$\beta$-actin & NM_001101 & $\begin{array}{l}\text { Forward } \\
\text { Reverse }\end{array}$ & 838 & $\begin{array}{l}\text { ATCTGGCACCACACCTTCTACAATGAGCTGCG } \\
\text { CGTCATACTCCTGCTTGCTGATTCACATCTGC }\end{array}$ \\
\hline
\end{tabular}

\section{Conclusions}

Polypyrrole has been successfully incorporated into a collagen-based PEC fiber in a bid to create a biofunctional, electroactive fiber system for tissue engineering. hMSCs cultured on these fibers expressed markers that are characteristic of neural lineage. Providing an external electrical stimulus to the culture system prompted the early expression of MAP2 and upregulation of synaptophysin. However, prolonged stimulation of the culture system proved detrimental. Thus, optimizing the electrical stimulation process would be necessary to prevent loss of hMSC viability. The interplay of a 3D architecture, fibrous topographical cue, and electroactivity should prove attractive for the creation of a biomimetic microenvironment to facilitate the differentiation of inducible stem cells to the neuronal lineage.

\section{Acknowledgements}

The authors would like to acknowledge financial support from NUS Research Scholarship (S.Z. Yow), Duke-NUS Graduate Medical School, the NUS Life Sciences Institute, and NIH-HL83008. 


\section{References}

1. Bieberich, E.; Anthony, G.E. Neuronal differentiation and synapse formation of PC12 and embryonic stem cells on interdigitated microelectrode arrays: Contact structures for neuron-to-electrode signal transmission (NEST). Biosens. Bioelectron. 2004, 19, 923-31.

2. Buzanska, L.; Zychowicz, M.; Ruiz, A.; Ceriotti, L.; Coecke, S.; Rauscher, H.; Sobanski, T.; Whelan, M.; Domanska-Janik, K.; Colpo, P.; Rossi, F. Neural stem cells from human cord blood on bioengineered surfaces-Novel approach to multiparameter bio-tests. Toxicology 2010, 270, $35-42$.

3. Haider, M.; Cappello, J.; Ghandehari, H.; Leong, K.W. In vitro chondrogenesis of mesenchymal stem cells in recombinant silk-elastinlike hydrogels. Pharm. Res. 2008, 25, 692-699.

4. Hwang, N.S.; Varghese, S.; Zhang, Z.; Elisseeff, J. Chondrogenic differentiation of human embryonic stem cell-derived cells in arginine-glycine-aspartate-modified hydrogels. Tissue Eng. 2006, 12, 2695-2706.

5. Yim, E.K.F.; Pang, S.W.; Leong, K.W. Synthetic nanostructures inducing differentiation of human mesenchymal stem cells into neuronal lineage. Experiment. Cell Res. 2007, 313, 1820-1829.

6. Engler, A.J.; Sen, S.; Sweeney, H.L.; Discher, D.E. Matrix elasticity directs stem cell lineage specification. Cell 2006, 126, 677-689.

7. Yow, S.Z.; Quek, C.H.; Yim, E.K.; Lim, C.T.; Leong, K.W. Collagen-based fibrous scaffold for spatial organization of encapsulated and seeded human mesenchymal stem cells. Biomaterials 2009, 30, 1133-1142.

8. Castano, H.; O'Rear, E.A.; McFetridge, P.S.; Sikavitsas, V.I. Polypyrrole thin films formed by admicellar polymerization support the osteogenic differentiation of mesenchymal stem cells. Macromol. Biosci. 2004, 4, 785-794.

9. Gomez, N.; Schmidt, C.E. Nerve growth factor-immobilized polypyrrole: bioactive electrically conducting polymer for enhanced neurite extension. J. Biomed. Mater. Res. A 2007, 81, 135-149.

10. Schmidt, C.E.; Shastri, V.R.; Vacanti, J.P.; Langer, R. Stimulation of neurite outgrowth using an electrically conducting polymer. Proc. Natl. Acad. Sci. USA 1997, 94, 8948-8953.

11. Guimard, N.K.; Gomez, N.; Schmidt, C.E. Conducting polymers in biomedical engineering. Progr. Polym. Sci. 2007, 32, 876-921.

12. Ateh, D.D.; Vadgama, P.; Navsaria, H.A. Culture of human keratinocytes on polypyrrole-based conducting polymers. Tissue Eng. 2006, 12, 645-655.

13. Au, H.T.; Cheng, I.; Chowdhury, M.F.; Radisic, M. Interactive effects of surface topography and pulsatile electrical field stimulation on orientation and elongation of fibroblasts and cardiomyocytes. Biomaterials 2007, 28, 4277-4293.

14. Cooper, S.T.; Maxwell, A.L.; Kizana, E.; Ghoddusi, M.; Hardeman, E.C.; Alexander, I.E.; Allen, D.G.; North, K.N. C2C12 Co-culture on a fibroblast substratum enables sustained survival of contractile, highly differentiated myotubes with peripheral nuclei and adult fast myosin expression. Cell Motility Cytoskel. 2004, 58, 200-211. 
15. Genovese, J.A.; Spadaccio, C.; Langer, J.; Habe, J.; Jackson, J.; Patel, A.N. Electrostimulation induces cardiomyocyte predifferentiation of fibroblasts. Biochem. Biophys. Res. Commun. 2008, 370, 450-455.

16. Green, R.A.; Lovell, N.H.; Wallace, G.G.; Poole-Warren, L.A. Conducting polymers for neural interfaces: Challenges in developing an effective long-term implant. Biomaterials 2008, 29, 3393-3399.

17. Park, H.; Bhalla, R.; Saigal, R.; Radisic, M.; Watson, N.; Langer, R.; Vunjak-Novakovic, G. Effects of electrical stimulation in C2C12 muscle constructs. J. Tissue Eng. Regen. Med. 2008, 2 , 279-287.

18. Pedrotty, D.M.; Koh, J.; Davis, B.H.; Taylor, D.A.; Wolf, P.; Niklason, L.E. Engineering skeletal myoblasts: roles of three-dimensional culture and electrical stimulation. Am. J. Physiol. Heart Circ. Physiol. 2005, 288, H1620-H1626.

19. Radisic, M.; Park, H.; Shing, H.; Consi, T.; Schoen, F.J.; Langer, R.; Freed, L.E.; Vunjak-Novakovic, G. Functional assembly of engineered myocardium by electrical stimulation of cardiac myocytes cultured on scaffolds. Proc. Natl. Acad. Sci. USA 2004, 101, 18129-18134.

20. Stern-Straeter, J.; Bach, A.D.; Stangenberg, L.; Foerster, V.T.; Horch, R.E.; Stark, G.B.; Beier, J.P. Impact of electrical stimulation on three-dimensional myoblast cultures - a real-time RT-PCR study. J. Cell. Mol. Med. 2005, 9, 883-892.

21. Sun, S.; Wise, J.; Cho, M. Human fibroblast migration in three-dimensional collagen gel in response to noninvasive electrical stimulus. I. Characterization of induced three-dimensional cell movement. Tissue Eng. 2004, 10, 1548-1557.

22. Gomez, N.; Lee, J.Y.; Nickels, J.D.; Schmidt, C.E. Micropatterned polypyrrole: A combination of electrical and topographical characteristics for the stimulation of cells. Adv. Func. Mater. 2007, $17,1645-1653$.

23. Wang, X.; Gu, X.; Yuan, C.; Chen, S.; Zhang, P.; Zhang, T.; Yao, J.; Chen, F.; Chen, G. Evaluation of biocompatibility of polypyrrole in vitro and in vivo. J. Biomed. Mater. Res. A 2004, 68, 411-22.

24. Garner, B.; Hodgson, A.J.; Wallace, G.G.; Underwood, P.A. Human endothelial cell attachment to and growth on polypyrrole-heparin is vitronectin dependent. J. Mater. Sci. Mater. Med. 1999, 10, 19-27.

25. Kotwal, A.; Schmidt, C.E. Electrical stimulation alters protein adsorption and nerve cell interactions with electrically conducting biomaterials. Biomaterials 2001, 22, 1055-1064.

26. Glowacki, J.; Mizuno, S. Collagen scaffolds for tissue engineering. Biopolymers 2008, 89, 338-344.

27. Friess, W. Collagen-biomaterial for drug delivery. Eur. J. Pharm. Biopharm. 1998, 45, 113-136.

28. Wang, Z.; Roberge, C.; Dao, L.H.; Wan, Y.; Shi, G.; Rouabhia, M.; Guidoin, R.; Zhang, Z. In vivo evaluation of a novel electrically conductive polypyrrole/poly(D,L-lactide) composite and polypyrrole-coated poly(D,L-lactide-co-glycolide) membranes. J. Biomed. Mater. Res. A 2004, 70, 28-38.

29. Li, H.C.; Khor, E. Interaction of collagen with polypyrrole in the production of hybrid materials. Polym. Int. 1994, 35, 53-59.

30. Ma, P.X. Biomimetic materials for tissue engineering. Adv. Drug Deliv. Rev. 2008, 60, 184-198. 
31. Wan, A.C.A.; Yim, E.K.F.; Liao, I.C.; Le Visage, C.; Leong, K.W. Encapsulation of biologics in self-assembled fibers as biostructural units for tissue engineering. J. Biomed. Mater. Res. A 2004, $71,586-595$.

32. Wan, A.C.A.; Liao, I.C.; Yim, E.K.F.; Leong, K.W. Mechanism of fiber formation by interfacial polyelectrolyte complexation. Macromolecules 2004, 37, 7019-7025.

33. Leroy-Lechat, F.; Wouessidjewe, D.; Andreux, J.-P.; Puisieux, F.; Duchêne, D. Evaluation of the cytotoxicity of cyclodextrins and hydroxypropylated derivatives. Int. J. Pharm. 1994, 101, 97-103.

34. Storsberg, J.; Ritter, H.; Pielartzik, H.; Groenendaal, L. Cyclodextrins in polymer synthesis: Supramolecular cyclodextrin complexes of pyrrole and 3,4-ethylenedioxythiophene and their oxidative polymerization. Adv. Mater. 2000, 12, 567-569.

(C) 2011 by the authors; licensee MDPI, Basel, Switzerland. This article is an open access article distributed under the terms and conditions of the Creative Commons Attribution license (http://creativecommons.org/licenses/by/3.0/). 